\title{
Design of a kind of automatic leveling device for crawler tractors Xiao Wang ${ }^{1, a}$, Xiaorong $\mathrm{Lv}^{1, \mathrm{~b}^{*}} \mathrm{Lin}^{\mathrm{He}} \mathrm{e}^{1, \mathrm{c}}$ and Tengfei $\mathrm{Wu}^{1, \mathrm{~d}}$ \\ ${ }^{1}$ Sichuan Agricultural Uniersity, 46 xinkang road, Ya'an ,Sichuan, China a934940773@qq.com, bIxrxj2008@163.com, ${ }^{\text {'5 } 572942740 @ q q . c o m,{ }^{d} 1484671243 @ q q . c o m}$ \\ "The Correspondence Author
}

Keywords: Crawler tractor; Leveling; Electric cylinder; Steering gear

\begin{abstract}
In order to improve the passability and stability of agricultural crawler tractors in different terrains in hilly and mountainous areas, and to improve the work quality of the agricultural machinery attached to it, this paper designed a track level automatic leveling device for crawler tractors in hilly areas. The device is characterized in that two electric cylinders are respectively arranged on the two sides of the tractor chassis as support, the movement of the vehicle body in the left and right direction is adjusted,the steering gear is connected to the body to limit the front and rear direction movement of the body. By adjusting the angle of rotation of the steering gear and the telescopic height of the electric cylinder to adjust the spatial angle of the platform, the purpose of keeping the tractor body level during the movement is maintained.
\end{abstract}

\section{Introduction}

The "13th Five-Year plan" proposed to vigorously promote agricultural modernization, the development of agricultural mechanization is an important symbol of modern agriculture ${ }^{[1]}$. Because the hilly and mountainous areas have the characteristics of uneven terrain, small fields, irregular, steep slope and many other characteristics, it is not conducive to the mechanization of field work, which hinders the development of Agricultural Mechanization in Hilly and mountainous $\operatorname{areas}^{[3] \sim[5]}$.

At present, domestic and foreign experts have conducted a lot of research on the leveling of the body, each with its own advantages and disadvantages ${ }^{[6] \sim[11]}$.In order to improve the working ability of agricultural tractors in hilly and mountainous areas, this paper designed a kind of automatic leveling device for crawler tractor body in hilly mountainous areas according to the topographical features of hilly mountainous areas, so that the tractor can maintain the level of the body when driving on different roads in hilly mountains. Which could increase the stability and trafficability of tractors and the ability of agricultural machinery to perform fine operations.

\section{Overall Structure Design and Working Principle of Automatic Leveling Device for Crawler Tractors}

Overall structure design. The tracked tractor studied in this paper is mainly composed of three parts, which are mainly divided into walking mechanism, leveling mechanism and measurement and control system, as shown in Figure 1. The leveling mechanism can adjust the body according to the instruction issued by the measurement and control system, so that the leveling auxiliary chassis can keep the level of the level on the pavement of different conditions so as to achieve the purpose of automatic leveling of the body. 


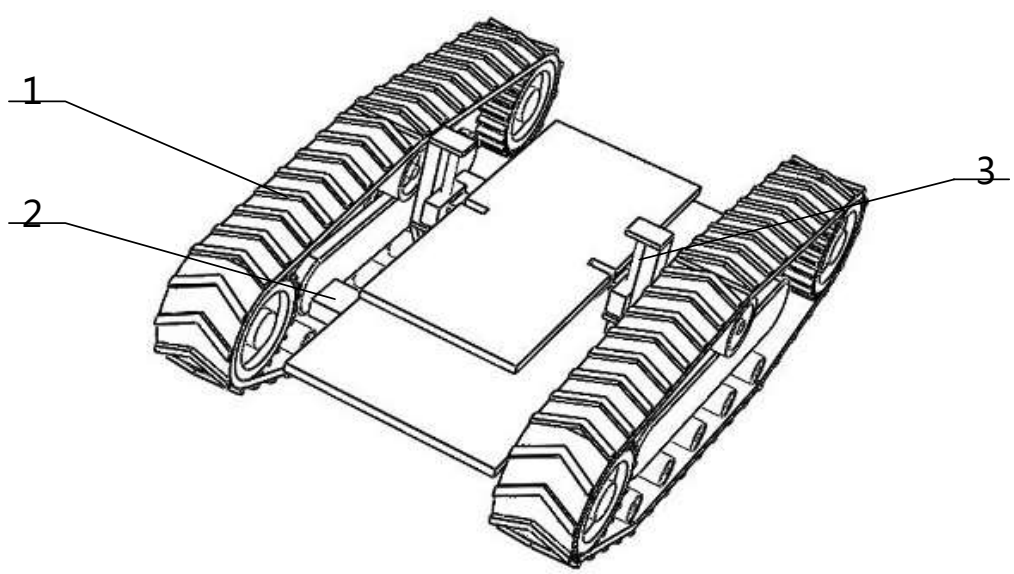

Figure 1. overall structure diagram

1. walking mechanism 2. measurement and control system 3. leveling mechanism

Working principle. When the tractors walk on the slope of the longitudinal slope, the leveling mechanism and the walking mechanism are in the tilted state before the leveling is not adjusted. The sensor detects the inclination of the body in the measurement and control system, and makes the leveling instructions through the calculation and analysis of the collected data through CPU, and controls the leveling operation of the leveling machine until the upper secondary chassis reaches the level. The body of the tractor designed this time is placed on the leveling sub-chassis, and the body is always level by the adjustment of the leveling mechanism.

\section{Main Structure Design and Working Principle of Leveling Mechanism}

Main structure design. In this design, the main mechanism of the automatic leveling device is the leveling mechanism, as shown in Figure 2 below. The leveling mechanism is mainly composed by electric cylinder, steering gear, three-headed cross shaft and a leveling sub-chassis. The electric cylinders are arranged on the left and right sides of the crawler tractor walking chassis, and support the leveling sub-chassis. The steering gears are arranged on the electric cylinder and can move up and down with the movement of the electric cylinders. The steering geasr are distributed on the left and right sides of the leveling sub-chassis and is connected to the leveling sub-chassis through three-headed cross shaft.

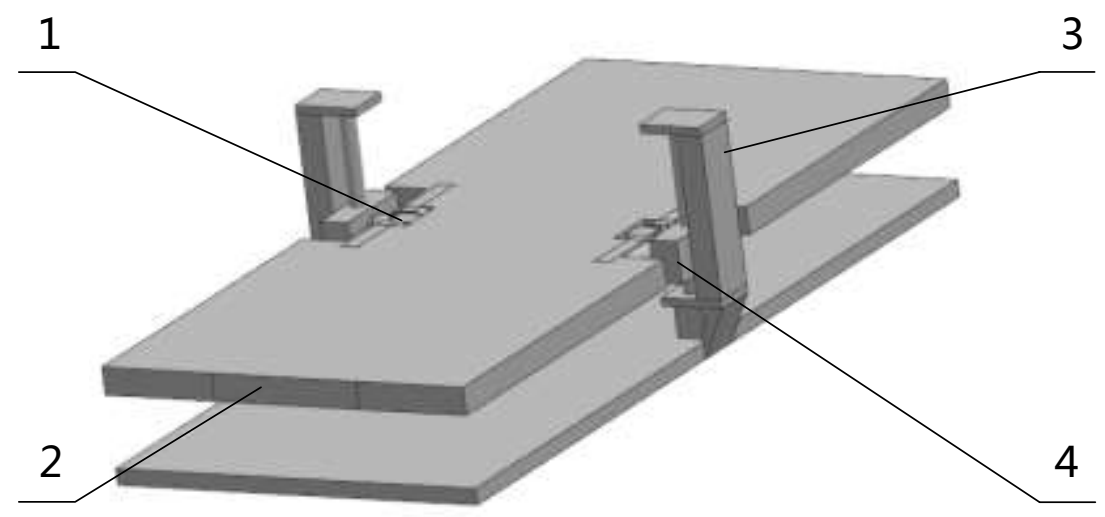

Figure 2. Structure diagram of leveling mechanism

1. three head cross shair 2 . leveling sub chassis 3 . electric cylinder 4. steering gear. 
Working principle. When the crawler tractor travels on the longitudinal ramp, the leveling sub chassis is tilted before it is leveled. At this time, the measurement and control system controls the steering gears, the output shaft of the steering gears drives the leveling sub chassis through a three cross shaft to turn a certain angle, so that the front and rear directions of the leveling sub chassis are in a horizontal state. At the same time, the measurement and control system controls the expansion of the electric cylinder so as to drive the leveling sub chassis to be raised or lowered horizontally, so as to maintain the level of the platform body. Through the combination of the steering gear and the electric cylinder, the leveling sub chassis can be adjusted in many ways.

\section{Design of measurement and control system}

The signal of measurement and control system in this design is mainly the inclination signal of the car. The signal is detected by the tilt sensor. The detected signal is analyzed and processed by the single chip microcomputer, and the response and output signal is made to control the expansion of the electric cylinder to control the movement of the leveling device, so as to realizes real-time monitoring of car body tilt and realtime leveling. At the same time, the LCD display can display the current angle in real time. The schematic diagram of the measurement and control system is shown in Figure 3 below.

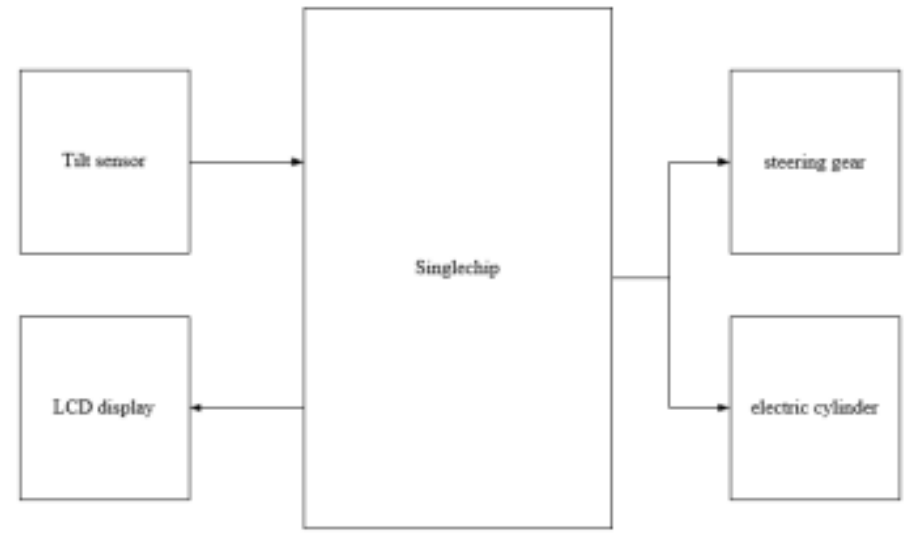

Figure 3. Schematic diagram of measurement and control system

The control strategy of the leveling of this car is mainly the angle compensation control leveling method. The body automatic leveling control system is mainly based on the body automatic leveling of the tilt sensor. The workflow is that when the automatic leveling is performed, the CPU receives the tilt signal after filtering by the tilt sensor, and then processes the leveling strategy after the automatic leveling algorithm is processed, the tilt signal is transmitted in real time, and finally the electromagnetic control is performed. The opening and closing of the valve, in turn, controls the action of the corresponding electric cylinder to level the body until the tilt sensor signal reaches a level.

\section{Working characteristics of automatic leveling device for crawler tractor}

The design of the crawler tractor is characterized in that the tilt sensor detects the tilt signal in real time while the body is running, and the processor processes the signal of the tilt sensor in real time, and adjusts the adjustment sub-chassis by controlling the movement of the electric cylinder and the steering gear. The real-time tilting condition achieves the purpose of real-time leveling. According to the characteristics of the road conditions in the hilly mountainous area and the tilting condition of the vehicle body, the telescopic length of the electric cylinder and the rotation angle of the steering gear are controlled, which effectively achieves the automatic leveling of the vehicle body during the walking of the tractor. 


\section{Acknowledgements}

The study was supported by National Natural Science Foundation of China (51105261): basic research on the application of general chassis steering system for planting technology.

\section{Summary}

Based on the tilt angle of the vehicle body, this paper designs a crawler tractor body automatic leveling device, which has simple and novel adjustment mechanism, small footprint, light weight, low energy consumption and quick adjustment. It can effectively increase the passability and stability of the crawler tractor and improve the quality and efficiency of the supporting small-scale agricultural implements.

This design adopts electric cylinder and steering gear to adjust the attitude of the vehicle body, energy consumption is small, and the electric cylinder and the steering gear can be self-locking, so that multi-directional and multi-angle simultaneous leveling can be carried out under the premise of stable body, and the adjustment is made. The leveling process is fast and stable.

\section{References}

[1] General Office of the State.Opinions of the State Council on Promoting the Sound and Rapid Development of Agricultural Mechanization and Agricultural Machinery Industry(Document of State Council[2012] No.22)[Z].2010-07-09

[2] General Office of the State. State Council's Opinions on Accelerating the Transformation of Agricultural Development Mode(Document of State Council[2015] No.59)[Z].2015-08-07

[3] General Office of the State.National Medium-and Long-Term Science and Technology Development Plan(Document of State Council[2006] No.6)[Z].2006-02-07

[4] General Office of the State.Made in China 2025(Document of State Council[2015] No.28)[Z].2015-05-08

[5] Xiaoliang Wang. Revo small tractor built for hilly mountains[J]. Agricultural machinery technology promotion, 2013,12:52

[6] Hengpei Liu.Influence of differential height mechanism on the stability of micro-track mountain tractor[J]. Tractor and agricultural transport vehicle,2013(1):18-21

[7] Lian $\mathrm{Hu}$, Chaoxing Lin, Xiwen Luo et al.Design and experiment of automatic leveling control system for agricultural machinery[J].Transactions of the Chinese Society of Agricultural Engineering,2015,31(8):15-17.

[8] Kai Liu, Lihong Yao, Qiang Zhang et al.Design of automatic leveling system for small-scale agricultural work vehicles[J].Jiangsu Agricultural Sciences,2013,41(3):363-365.

[9] Ramesh Kumar P, Asif Chalanga, B. Bandyopadhyay Smooth integral sliding mode controller for the position control of Stewart platform[J]. IS A Transactions 2015,(58): 543-551.

[10] Sandipan Bandyopadhyay, Ashitava Ghosal, An algebraic formulation of kinematic isotropy and design of isotropic 6-6 Stewart platform manipulators[J]. Mechanism and Machine Theory.2008(43):591-616.

[11] Kai Peng, Ping Jiang, Wenwu Hu et al. Development and experiment of leveling mechanism for three-point suspension machine of rotary tiller[J].Journal of Hunan Agricultural University,2015,41(6):676-679. 